EUROPEAN ORGANIZATION FOR NUCLEAR RESEARCH

CERN-EP/99-74

5 May 1999

\title{
Direct detection of Earth matter effects (MSW) in flavor oscillations at neutrino beams from stored muon decays
}

\author{
A. Bueno ${ }^{1}$, M. Campanelli ${ }^{1}$, A. Rubbia ${ }^{1,2}$ \\ ${ }^{1}$ Institut für Teilchenphysik, ETHZ, CH-8093 Zürich, Switzerland \\ ${ }^{2}$ CERN, Geneva, Switzerland
}

\begin{abstract}
We explore the possibility of a neutrino oscillation experiment with a very long baseline in the range of $6500 \mathrm{~km}$ and a neutrino beam produced by the decays of muons circulating in a storage ring. The recent developments in view of muon colliders allow us to envisage neutrino sources of a sufficiently high intensity. We first consider $\nu_{e} \leftrightarrow \nu_{\mu}$ oscillations within a three flavor oscillation framework. Evidence for this oscillation implies that the (1-3) mixing is non-zero. We study the effect of the neutrino propagation through matter (MSW effect). Given the density of the Earth, the existence of the MSW resonance can be experimentally proven by comparing the oscillated spectra of neutrinos obtained from the decays of muons of positive and negative charges. Moreover, a precision study of the oscillations of all three flavors could be performed since neutrinos are above the tau production threshold (appearance searches).
\end{abstract}




\section{Introduction}

As well known, neutrino flavor oscillations will take place if the neutrino flavor eigenstates $\left(\nu_{e}, \nu_{\mu}\right.$ and $\left.\nu_{\tau}\right)$ do not coincide with their mass eigenstates $\left(\nu_{1}, \nu_{2}\right.$ and $\left.\nu_{3}\right)$. Within the two flavor oscillation framework, the transition probability in vacuum between flavor $\nu_{\alpha}$ and $\nu_{\beta}(\alpha, \beta=e, \mu, \tau, \alpha \neq \beta)$ is given by:

$$
P\left(\nu_{\alpha} \rightarrow \nu_{\beta}\right)=\sin ^{2} 2 \theta \sin ^{2}\left(1.27 \Delta m_{i j}^{2}\left(e V^{2}\right) \frac{L(k m)}{E_{\nu}(G e V)}\right)
$$

where $\Delta m_{i j}^{2}=m_{i}^{2}-m_{j}^{2}(i, j=1,2,3, i \neq j)$ is the mass squared difference between the two neutrino mass eigenstates, $\nu_{i}$ and $\nu_{j}, \theta$ is the mixing angle between mass and weak eigenstates, $L$ is the baseline, and $E_{\nu}$ the neutrino energy. When the neutrinos propagate in matter, their equation of motion must include an interaction with the medium. For a given neutrino energy which depends on the matter electron density, a "resonance" condition will be reached (the MSW-effect[1]) at which the flavor oscillation of neutrinos (antineutrinos) will then be enhanced (suppressed) compared to oscillations in vacuum.

The results on the solar and atmospheric neutrino fluxes can be naturally explained in terms of neutrino oscillations. Since the energies and distances of the solar neutrino problem are widely different from those of the atmospheric neutrino anomaly, the neutrino oscillation solutions require very different values of mass squared differences.

The solar neutrino deficit[2] can be explained via the disappearance of $\nu_{e}$ 's due to flavor oscillation enhanced by the MSW effect. A combined fit to the experimental data implies a $\Delta m^{2}$ in the region $10^{-5} \mathrm{eV}^{2}$ and two possible angular solutions: the small (SMA) and large (LMA) mixing angle solutions. Another solution to these data would require a $\Delta m^{2} \approx 10^{-10} \mathrm{eV}^{2}$ if the oscillation took place in vacuum.

The MSW solution of the solar neutrino problem is the more attractive one because it does not require the fine-tuning of the oscillation parameters. The conversion in the Sun is primarily a resonance phenomenon, which occurs at a specific density that corresponds to a definite neutrino energy (for a given $\Delta m^{2}$ ). Because at night solar neutrinos will cross the Earth before reaching the detector, the MSW mechanism may introduce a day-night asymmetry[10] by the phenomenon of "regeneration". However, in the relatively small density of the Earth, the resonance condition is not met. Clearly the search for the MSW effect on Earth will be of great importance.

The observation of atmospheric neutrinos by SuperKamiokande[3] and other detectors[4] (Kamiokande, IMB, Soudan-II and MACRO) has shown evidence for neutrino flavor oscillations, compatible with $\nu_{\mu}$ neutrinos converting to $\nu_{\tau}$ with maximal mixing and $10^{-3} \lesssim \Delta m^{2} \lesssim 10^{-2} \mathrm{eV}^{2}$

For the present generation of long baseline beams from CERN and Fermilab, the baseline of $L=730 \mathrm{~km}$ is too short to provide a strong resonance signal[5]. Within this context, we explore the possibility of an experiment using neutrinos from stored muon decays at a very long baseline of $6500 \mathrm{~km}$, with neutrinos of sufficient energy in order to produce the resonance phenomena in their passage through Earth for the set of parameters suggested by the atmospheric neutrino results. Since this phenomenology implies oscillations into tau neutrinos, the energy should also be large enough in order to observe explicitely the charged current interactions of $\nu_{\tau}$ 's.

The synergy with future muon colliders[6] is here twofold: Muon colliders will nec- 
essarily require very intense proton sources and it will be then concevable to obtain very intense neutrino beams[7] to compensate for the flux decrease with increasing distance from the source. In addition, the well-defined flavor composition of beams from muon decays can be exploited using a detector with charge identification capabilities to look for flavor oscillations[8].

We first consider $\nu_{e} \leftrightarrow \nu_{\mu}$ oscillations within a three flavor oscillation framework, at the $\Delta m_{32}^{2}=m_{3}^{2}-m_{2}^{2}$ mass indicated by the atmospheric neutrinos. Evidence for this oscillation would imply that the (1-3) mixing between the first and the third family is non-zero. We study the effect of the neutrino propagation through the matter of the Earth (MSW effect). Given the density of the Earth, the MSW resonance occur at high energies accessible with accelerators.

With the use neutrino beams from stored muon decays, the matter enhancement or suppression of the oscillation can be experimentally tested by comparing the event rates and energy spectra obtained from decaying stored muons of positive and negative charges.

We then consider the study of oscillations of all three flavors. Our high energy neutrino beam is well above tau threshold, therefore opening the channel of tau appearance searches. These would be optimized for the $\Delta m^{2}$ mass indicated by the atmospheric neutrino anomaly since the baseline is such that the relevant parameter $E_{\nu} / L$ is exactly in that range of $\Delta m^{2}$. The transition probabilities will therefore be maximized and the flavor oscillation pattern will be visible as a function of the incoming neutrino energy. This is a main difference with respect to presently planned long baseline beams where given the baseline of $730 \mathrm{~km}$ the basic oscillation can be measured only in the upper part of the region indicated by the atmospheric neutrinos. Extending the baseline without a loss of rate will enlarge the explored $\Delta m^{2}$ region correspondingly.

\section{$2 \quad$ Neutrinos from decays of stored muons}

The neutrino beams from the decays of stored muon[7] provide an ideal configuation for the study of matter effects. First of all,

- they will contain neutrinos of the electron and muon flavors in same quantity of a well-defined helicity composition depending on the muon charge, i.e. $\mu^{-} \rightarrow e^{-} \overline{\nu_{e}} \nu_{\mu}$ or $\mu^{+} \rightarrow e^{+} \nu_{e} \bar{\nu}_{\mu}$.

- the charge of the muon can be easily selected, ideally within each fillings of the storage ring.

These features distinguish them from traditional neutrino beams where $\nu_{\mu}$ dominates, where $\nu_{e}$ comes from kaon decays since highly suppressed in $\pi \rightarrow e \nu_{e}$, and in which the neutrino-antineutrino configurations are not symmetric. In addition, the fluxes of neutrino beams from muon decays can be easily predicted (since no hadronic processes involved) when the muon polarization is known and are flexible in the choice of the beam energy, since precisely determined by the muon storage ring energy.

Secondly, the well-defined flavor composition can be exploited using a detector with charge identification capabilities (see Ref. [8]). For decays of negative stored muons $\left(\mu^{-} \rightarrow e^{-} \overline{\nu_{e}} \nu_{\mu}\right)$, the charged current neutrino interactions will produce leading electrons of positive charge $\left(\overline{\nu_{e}} N \rightarrow e^{+}+X\right)$ and leading muons of negative charge $\left(\nu_{\mu} N \rightarrow \mu^{-}+X\right)$. In case of neutrino $\nu_{e} \leftrightarrow \nu_{\mu}$ oscillations, there will be appearance of negative leading electrons $\nu_{\mu} \rightarrow \nu_{e} N \rightarrow e^{-}+X$ and of positive leading muons $\bar{\nu}_{e} \rightarrow \bar{\nu}_{\mu} N \rightarrow \mu^{+}+X$. For 
decays of positive stored muons, the charge conjugate configurations will occur.

In the case of massive detectors, it will be easier to measure the charge of leading muons than that of electrons. The golden channel to look for oscillations is therefore provided by the search for leading muons of opposite sign of the stored muons. This will be discussed in section 5 . Some information will also be available from the leading electron sample without charge discrimination but with lesser sensitivity.

\section{$3 \quad$ Very long baselines and neutrino source requirements}

Since muon colliders necessarily require very intense proton sources, it will be possible to obtain very intense neutrino beams to perform long and very long baseline oscillation experiments $^{1)}$.

We assume here for the sake of a concrete example that the neutrino detector will be located in Europe at the Laboratori Nazionali del GranSasso (LNGS). The neutrino source could then be located in different laboratories around the world, in the American (BNL, FNAL) or Asian (KEK) continent.

The BNL-LNGS has a baseline of $L=6500 \mathrm{~km}$ (see Figure 1). The beam goes to a maximum depth of $900 \mathrm{~km}$ and arrives at GS with an angle with respect to horizontal of about $30^{\circ}$. We estimate the average density of the Earth[10] for this baseline to be $\rho=3.6 \mathrm{~g} / \mathrm{cm}^{3}$. Other baselines are FNAL-GranSasso $(L=7400 \mathrm{~km}$, max. depth 1200 $\mathrm{km}$, average density $\rho=3.7 \mathrm{~g} / \mathrm{cm}^{3}$, beam angle $\left.36^{\circ}\right)$ or KEK-GranSasso $(L=8800 \mathrm{~km}$, max. depth $1800 \mathrm{~km}$, average density $\rho=4.0 \mathrm{~g} / \mathrm{cm}^{3}$, beam angle $44^{\circ}$ ). There is not much differences in $L$ between the various baselines.

In a neutrino beam from stored decaying muons, the neutrino event rate will grow as $E_{\mu}^{3}$ where $E_{\mu}$ is the energy of the muon storage ring. We are interested in a neutrino beam with energy in the range of $10-30 \mathrm{GeV}$. We list in Table 1 the neutrino event rates in case of no oscillation as a function of the muon storage ring energy, for $10^{21}$ muon decays of a given charge and 10 kton target. Neutrino interactions have been divided into charged current (CC) and neutral current (NC) interactions. The CC events include inelastic scattering (DIS) and quasi-elastic (QE) interactions.

To reach $10^{21}$ muon decays, we refer to recent simulations performed for muon colliders studies[6] which consider a scheme in which the yield of accumulated muons in the storage ring per $16 \mathrm{GeV}$ proton impinging on the primary target is about $10-15 \%$.

Such a storage ring would be operated for four years, alternating runs with positive and negative muons. Given the shape of the muon storage ring, about $40 \%$ of the muons would decay in the direction towards the neutrino detector (the rest decays in the bending section and in the opposite direction). To achieve an integrated intensity a total of

$$
N\left(\mu^{+}\right)+N\left(\mu^{-}\right)=N_{p} \times 0.15 \times 0.4 \times 4 \simeq 2 \times 10^{21} \mu^{\prime} \mathrm{s}
$$

requires a proton source of the order of $N_{p} \simeq 10^{22}$ protons/year. An upgrade of the AGS accelerator in BNL[11] could yield an integrated intensity close to this figure.

1) In Ref. [9], we have considered a $\nu_{\mu}$ disappearance experiment with a baseline of $730 \mathrm{~km}$. Since the neutrino beams contain both electron and muon flavors, the disappearance of muon neutrino can be performed by directly comparing electron and muon events. This method is self normalizing, i.e. without the need of a near detector to predict the original flux. 


\begin{tabular}{ccccccc}
\hline & \multicolumn{3}{c}{$10^{21} \mu^{-}$decays } & \multicolumn{3}{c}{$10^{21} \mu^{+}$decays } \\
$E_{\mu}(\mathrm{GeV})$ & $\mathrm{CC}\left(\bar{\nu}_{e}\right)$ & $\mathrm{CC}\left(\nu_{\mu}\right)$ & $\mathrm{NC}$ & $\mathrm{CC}\left(\nu_{\boldsymbol{e}}\right)$ & $\mathrm{CC}\left(\bar{\nu}_{\mu}\right)$ & $\mathrm{NC}$ \\
\hline \hline 10 & 426 & 1152 & 196 & 1016 & 488 & 173 \\
15 & 1414 & 3751 & 1129 & 3283 & 1624 & 1005 \\
20 & 3313 & 8712 & 3542 & 7588 & 3820 & 3172 \\
25 & 6412 & 16746 & 8221 & 14568 & 7401 & 7419 \\
30 & 11010 & 28576 & 16008 & 24850 & 12710 & 14524 \\
\hline
\end{tabular}

Table 1: The total number of neutrinos detected in a 10 kton (fiducial) detector for a baseline $L=6500 \mathrm{~km}$ and a total number of $10^{21}$ muons decays.

\section{$4 \quad$ Matter enhanced neutrino oscillations}

In three-family scenarios[12], the mixing between the neutrino flavors will be determined by a $3 \times 3$ unitary matrix describing the mixing between flavor and mass eigenstates. With three neutrino states, oscillations will be determined by only two independent mass square differences, say $\Delta m_{32}^{2}=m_{3}^{2}-m_{2}^{2}$ and $\Delta m_{21}^{2}=m_{2}^{2}-m_{1}^{2}$.

The current experimental results on solar and atmospheric neutrinos allow us to consider the approximation that only one mass scale is relevant, since if we assign $\Delta m_{32}^{2}\left(\Delta m_{21}^{2}\right)$ to the atmospheric(solar) oscillations, then $\Delta m_{32}^{2} \gg \Delta m_{21}^{2}$. This implies that the two oscillations driven by the mass differences $\Delta m_{32}^{2}$ and $\Delta m_{21}^{2}$ decouple and can be studied independently.

The three-family oscillation is then described by only three parameters: the mass difference $\Delta m_{32}^{2}$ and the two mixing angles $\theta$ and $\phi$. The mass eigenstate $m_{1}$ is defined orthogonal to the electron flavor state. The mixing matrix takes then the form:

$$
U=\left(\begin{array}{ccc}
0 & \cos \theta & \sin \theta \\
\cos \phi & -\sin \theta \sin \phi & \cos \theta \sin \phi \\
-\sin \phi & -\sin \theta \cos \phi & \cos \theta \cos \phi
\end{array}\right)
$$

We assume that $\nu_{3}$ is the heaviest state and the atmospheric neutrino data implies that the mixing is maximal between $\nu_{\mu}$ and $\nu_{\tau}\left(\sin ^{2} \phi=\cos ^{2} \phi=0.5\right)$. The flavor oscillation probabilities for neutrinos and antineutrinos are then simply:

$P\left(\nu_{e} \rightarrow \nu_{\mu}\right)=P\left(\nu_{e} \rightarrow \nu_{\tau}\right)=P\left(\bar{\nu}_{e} \rightarrow \bar{\nu}_{\mu}\right)=P\left(\bar{\nu}_{e} \rightarrow \bar{\nu}_{\tau}\right)=\sin ^{2} 2 \theta \sin ^{2}\left(1.27 \Delta m_{32}^{2}\left(e V^{2}\right) \frac{L(k m)}{E_{\nu}(G e V)}\right)$

The negative result from CHOOZ[13] on electron disappearance and the SuperKamiokande data themselves constrain the mixing angle $\theta$. A value compatible with observation is $\sin ^{2} \theta=0.025[5]$.

In matter, the modification of the flavor transition is taken into account by the mixing angle in matter $\theta_{m}$, which is:

$$
\sin ^{2} 2 \theta_{m}^{\mp}(x)=\frac{\sin ^{2} 2 \theta}{\sin ^{2} 2 \theta+(x \mp \cos 2 \theta)^{2}}
$$


where the minus sign applies to $\nu$ 's and the plus to $\bar{\nu}$ 's and

$$
x=\frac{2 \sqrt{2} G_{F} n_{e} E_{\nu}}{\Delta m^{2}} \approx 0.76 \times 10^{-4} \frac{\rho\left(\mathrm{g} / \mathrm{cm}^{3}\right) E_{\nu}(\mathrm{GeV})}{\Delta m^{2}\left(e V^{2}\right)} .
$$

where $n_{e}$ is the electron density of the medium. The transition probabilities are then:

$$
\begin{aligned}
& P\left(\nu_{e} \rightarrow \nu_{\mu}\right)=P\left(\nu_{e} \rightarrow \nu_{\tau}\right)=\sin ^{2}\left(2 \theta_{m}^{-}\right) \sin ^{2}\left(1.27 \Delta m_{32}^{2}\left(e V^{2}\right) \frac{\lambda^{-}(k m)}{E_{\nu}(G e V)}\right) \\
& P\left(\bar{\nu}_{e} \rightarrow \bar{\nu}_{\mu}\right)=P\left(\bar{\nu}_{e} \rightarrow \bar{\nu}_{\tau}\right)=\sin ^{2}\left(2 \theta_{m}^{+}\right) \sin ^{2}\left(1.27 \Delta m_{32}^{2}\left(e V^{2}\right) \frac{\lambda^{+}(k m)}{E_{\nu}(G e V)}\right)
\end{aligned}
$$

where $\lambda^{ \pm}=L \times \sqrt{\sin ^{2} 2 \theta+(x \pm \cos 2 \theta)^{2}}$. For neutrinos, the resonance condition will be met when $x\left(E_{\nu}, \Delta m^{2}, \rho\right) \simeq \cos 2 \theta$ and the oscillation amplitude will reach a maximum. For the resonant neutrino energy, $E_{\nu}^{r e s}$, this reads

$$
E_{\nu}^{r e s} \approx \frac{1.32 \times 10^{4} \cos 2 \theta \Delta m^{2}\left(e V^{2}\right)}{\rho\left(g / \mathrm{cm}^{3}\right)} \approx 0.37 \times 10^{4} \Delta m^{2}\left(e V^{2}\right)
$$

where we have assumed a constant Earth density $\rho=3.6 \mathrm{~g} / \mathrm{cm}^{3}$ and small mixing angles. For the parameters indicated by the atmospheric neutrino observation $10^{-3} \lesssim \Delta m^{2} \lesssim$ $10^{-2} \mathrm{eV}^{2}$, we obtain $3.7 \lesssim E_{\nu}^{\text {res }} \lesssim 37 \mathrm{GeV}$, i.e. the resonance energy lies in the region accessible to high energy accelerator neutrino beams.

The conversion from $\nu_{\mu} \rightarrow \nu_{\tau}$ flavor is independent of matter effects and is given by the probability:

$$
P\left(\nu_{\mu} \rightarrow \nu_{\tau}\right)=P\left(\bar{\nu}_{\mu} \rightarrow \bar{\nu}_{\tau}\right) \approx \sin ^{2}\left(1.27 \Delta m_{32}^{2}\left(e V^{2}\right) \frac{L(k m)}{E_{\nu}(G e V)}\right)
$$

where we have made the approximation that $\cos ^{2} \theta=1$ since $\theta$ is a small angle. Given our choice of $E_{\nu} / L \approx \Delta m_{32}^{2}$, there will be large oscillation to $\nu_{\tau}\left(\bar{\nu}_{\tau}\right)$.

\section{Detection of Neutrino oscillations and matter effects}

We consider in more detail neutrino oscillations for $\Delta m^{2}=3 \times 10^{-3} \mathrm{eV}^{2}$. Our conclusion remain unchanged for values of $\Delta m^{2}$ in the range $10^{-2} \lesssim \Delta m^{2} \lesssim 10^{-3} \mathrm{eV}^{2}$ (see Ref. [14]). We study the three-family oscillation of the two neutrino flavors present in the beam. In the case of a neutrino beam from negative muons, we will observe $\nu_{\mu} \rightarrow \nu_{e}$, $\nu_{\mu} \rightarrow \nu_{\tau}$, and $\bar{\nu}_{e} \rightarrow \bar{\nu}_{\mu}, \bar{\nu}_{e} \rightarrow \bar{\nu}_{\tau}$ (for positive muons the charge-conjugate processes).

The oscillated neutrino fluxes as a function of energy for the different flavors are shown in Figure 2 for a $30 \mathrm{GeV}$ muon beam. The difference between the $\bar{\nu}_{e} \rightarrow \bar{\nu}_{\mu}$ in $\mu^{-}$ decays and the $\nu_{e} \rightarrow \nu_{\mu}$ in $\mu^{+}$decays is clearly visible. We observe a similar effect for $\nu_{e}\left(\bar{\nu}_{e}\right)$ appearance in $\mu^{-}\left(\mu^{+}\right)$decays. In both cases, the $\nu_{\mu} \rightarrow \nu_{\tau}\left(\bar{\nu}_{\mu} \rightarrow \bar{\nu}_{\tau}\right)$ conversions are maximal and correspondingly the $\nu_{\mu}\left(\bar{\nu}_{\mu}\right)$ are highly depleted. The neutrinos will be detected in their charged and neutral current interactions.

The detector should have excellent electron and muon identification and measurement capabilities. These lepton capabilities should be matched to the beams from muon decays, which provide equal amounts of electron and muon neutrinos. These features would 
be all met by a large detector based on liquid argon imaging technology (see ICARUS Ref [15] and references therein). We also require a charge-determination by means of a muon spectrometer, to allow the identification of the leading muon charge. On the other hand, the leading electron charge cannot be identified.

We classify the observed events in four classes:

a) events with electrons or positrons (no electron charge measured),

b) events with muons of the same sign of those circulating in the storage ring,

c) events with muons of opposite sign,

d) events without leptons.

In this study, we do not consider the possibility to directly identify appearance of $\nu_{\tau}$ neutrinos by means of a direct detection of the tau lepton in final states ${ }^{2}$. Therefore, according to the decay mode of the tau lepton, $\nu_{\tau}$ CC events are seen in the electron $(\tau \rightarrow e$ decays), muon $(\tau \rightarrow \mu)$ or neutral current $(\tau \rightarrow h)$ sample. Since for the parameters of the atmospheric neutrinos, the oscillation probabilities are large, a tau appearance signal appears as a clear excess of events, in particular in the neutral current like sample, which corresponds to the largest branching fraction of the tau.

In the following sections, we compute the number of events expected in each of the four classes for an integrated muon intensity of $10^{21}$ decays of each charge. For $\nu_{\tau}$ interactions, the tau lepton kinematical suppression has been taken into account and rates include quasi-elastic and deep-inelastic contributions. We will use the notation $P_{\nu_{\alpha} \rightarrow \nu_{\beta}}$ to identify the probability of neutrinos of flavor $\alpha$ to oscillate into neutrinos of flavor $\beta$.

\subsection{Events with opposite-sign muons}

The muon charge is measured by a spectrometer placed behind the detector. Oppositesign leading muons can only be produced by neutrino oscillations, since there is no component in the beam that could account for them ${ }^{3}$ ). These events are coming from oscillations of the electron component of the beam.

For the case of $\mu^{-}$decays, we have appearance of opposite-sign muons

1. directly via $\bar{\nu}_{e} \rightarrow \bar{\nu}_{\mu}$ oscillations

$P_{\bar{\nu}_{e} \rightarrow \bar{\nu}_{\mu}} \times \Phi_{\bar{\nu}_{e}}$

2. via $\tau$ decays after $\bar{\nu}_{e} \rightarrow \bar{\nu}_{\tau}$ oscillations.

$P_{\bar{\nu}_{e} \rightarrow \bar{\nu}_{\tau}} \times B R(\tau \rightarrow \mu) \times \Phi_{\bar{\nu}_{e}}$

These contributions are listed in Table 2 from decays of positive and negative muons.

The matter effect appears clearly. For beams from decays of $\mu^{-}\left(\mu^{+}\right)$, the oscillation is suppressed(enhanced). The effect is very striking for example for $E_{\mu}=30 \mathrm{GeV}$, where we expect 24 matter-suppressed $\mu^{+}$events in $\mu^{-}$decays against 1703 matter-enhanced $\mu^{-}$events in $\mu^{+}$decays. In addition, there will be $90 \nu_{e} \rightarrow \nu_{\tau}$ followed by $\tau^{-} \rightarrow \mu^{-}$in $\mu^{+}$ and $1 \bar{\nu}_{e} \rightarrow \bar{\nu}_{\tau}, \tau^{+} \rightarrow \mu^{+}$in $\mu^{-}$decays.

2) These can be achieved either by "topological" searches in which a decay kink is looked for or by "kinematical" methods in which the presence of unseen neutrinos in the final states is detected by an analysis of the final state kinematics

3) Opposite sign muon background comes from decays of hadrons in neutral current events and from charm decays. We note however that these muons will be soft and not isolated from the jet and can therefore be suppressed by a mild isolation and momentum cut. For charm produced in charged current events, the leading lepton should be misidentified. 


\begin{tabular}{ccccc}
\hline$E_{\mu}(\mathrm{GeV})$ & $\nu_{e} \rightarrow \nu_{\mu}$ & $\nu_{e} \rightarrow \nu_{\tau}$ & unoscillated beam & $\bar{\nu}_{\mu} \rightarrow \bar{\nu}_{\tau}$ \\
$\mu^{+}$decays & $\mu^{-}$ & $\tau^{-} \rightarrow \mu^{-}$ & $\mu^{+}$ & $\tau^{+} \rightarrow \mu^{+}$ \\
\hline 10 & 186 & 4 & 349 & 3 \\
20 & 1086 & 44 & 562 & 183 \\
30 & 1703 & 90 & 3484 & 655 \\
\hline$\mu^{-}$decays & $\bar{\nu}_{e} \rightarrow \bar{\nu}_{\mu}$ & $\bar{\nu}_{e} \rightarrow \bar{\nu}_{\tau}$ & unoscillated beam & $\nu_{\mu} \rightarrow \nu_{\tau}$ \\
& $\mu^{+}$ & $\tau^{+} \rightarrow \mu^{+}$ & $\mu^{-}$ & $\tau^{-} \rightarrow \mu^{-}$ \\
\hline 10 & 4 & 0 & 580 & 6 \\
20 & 22 & 1 & 1049 & 370 \\
30 & 24 & 1 & 7893 & 1390 \\
\hline
\end{tabular}

Table 2: Contributions to leading-muon events normalized to $10^{21} \mu$ decays of each sign for a stored muon energy of 10,20 and $30 \mathrm{GeV}$. The appearance of opposite-sign muons is enhanced(suppressed) by matter effects for decaying $\mu^{+}\left(\mu^{-}\right)$beams.

\subsection{Events with right-sign muons}

Leading muons of the same sign of those decaying in the storage ring are produced by:

1. unoscillated muon neutrinos coming from the beam $\left(1-P_{\nu_{\mu} \rightarrow \nu_{e}}-P_{\nu_{\mu} \rightarrow \nu_{\tau}}\right) \times \Phi_{\nu_{\mu}}$

2. $\tau$ decays, where the $\tau$ is coming from $\nu_{\mu} \rightarrow \nu_{\tau}$ oscillations.

$P_{\nu_{\mu} \rightarrow \nu_{\tau}} \times B R(\tau \rightarrow \mu) \times \Phi_{\nu \mu}$

The right-sign muon events are strongly depleted due to the maximal oscillation to $\nu_{\tau}$ 's. Given the proper $L / E_{\nu}$, the conversion to the tau flavor is maximized. The disappearance of right-sign muons will yield in the usual way the $\Delta m^{2}$ parameter. These oscillations are almost unaffected by matter because of the mixing angle $\sin \theta$ is much smaller than $\sin \phi$.

\subsection{Events with electrons}

Events with leading electron or positron are produced by the charged-current interactions of the following neutrinos:

1. unoscillated $\overline{\nu_{e}}$ neutrinos from the beam

$$
\left(1-P_{\bar{\nu}_{e} \rightarrow \bar{\nu}_{\mu}}-P_{\bar{\nu}_{e} \rightarrow \bar{\nu}_{\tau}}\right) \times \Phi_{\bar{\nu}_{e}}
$$

2. muon neutrinos oscillated into electron neutrinos

$$
P_{\nu_{\mu} \rightarrow \nu_{e}} \times \Phi_{\nu_{\mu}}
$$

3. tau neutrinos derived from oscillations followed by a $\tau \rightarrow e$ decay

$$
P_{\nu_{\mu} \rightarrow \nu_{\tau}} \times B R\left(\tau^{-} \rightarrow e\right) \times \Phi_{\nu_{\mu}}+P_{\bar{\nu}_{e} \rightarrow \bar{\nu}_{\tau}} \times B R\left(\tau^{+} \rightarrow e\right) \times \Phi_{\bar{\nu}_{e}}
$$

In case of $\mu^{-}$beams, process 1 ) will deplete the number of leading electron events from the beam, while process 2) will increase the number of such events which come from matterenhanced oscillations of muon neutrinos. The net effect is an increase of the number of observed events having a leading electron with respect to the case of no oscillation, as can be seen by comparing Table 3 with the non-oscillated case in Table 1. For example for $30 \mathrm{GeV} \mu^{-}$decays, in case of no oscillations, we expect $11010 \bar{\nu}_{e} \mathrm{CC}$ events against $10967\left(\bar{\nu}_{e}\right)+1366\left(\nu_{\mu} \rightarrow \nu_{e}\right) \mathrm{CC}$ in case of oscillations. For $\mu^{+}$decays, we have $24850 \nu_{e} \mathrm{CC}$ events against $21457\left(\nu_{e}\right)+15\left(\bar{\nu}_{\mu} \rightarrow \bar{\nu}_{e}\right)$ CC in case of oscillations. 


\begin{tabular}{cccc}
\hline$E_{\mu}(\mathrm{GeV})$ & unoscillated beam & $\nu_{\mu} \rightarrow \nu_{e}$ & $\tau$ decay \\
$\mu^{-}$decays & $\left(1-P_{\bar{\nu}_{e} \rightarrow \bar{\nu}_{x}}\right) \times \Phi_{\bar{\nu}_{e}}$ & $\left(P_{\nu_{\mu} \rightarrow \nu_{e}}\right) \times \Phi_{\nu_{\mu}}$ & $\left(\left(P_{\nu_{\mu} \rightarrow \nu_{\tau}}\right) \Phi_{\nu_{\mu}}+\left(P_{\bar{\nu}_{e} \rightarrow \bar{\nu}_{\tau}}\right) \Phi_{\bar{\nu}_{e}}\right) \times B R(\tau \rightarrow e)$ \\
\hline 10 & 418 & 252 & 6 \\
20 & 3268 & 977 & 380 \\
30 & 10967 & 1366 & 1423 \\
\hline & unoscillated beam & $\bar{\nu}_{\mu} \rightarrow \bar{\nu}_{e}$ & $\tau$ decay \\
$\mu^{+}$decays & $\left(1-P_{\nu_{e} \rightarrow \nu_{x}}\right) \times \Phi_{\nu_{e}}$ & $\left(P_{\bar{\nu}_{\mu} \rightarrow \bar{\nu}_{e}}\right) \times \Phi_{\bar{\nu}_{\mu}}$ & $\left(\left(P_{\bar{\nu}_{\mu} \rightarrow \bar{\nu}_{\tau}}\right) \Phi_{\bar{\nu}_{\mu}}+\left(P_{\nu_{e} \rightarrow \nu_{\tau}}\right) \Phi_{\nu_{e}}\right) \times B R(\tau \rightarrow e)$ \\
\hline 10 & 644 & 5 & 7 \\
20 & 5413 & 17 & 233 \\
30 & 21457 & 15 & 761 \\
\hline
\end{tabular}

Table 3: Contributions to leading electron events normalized to $10^{21} \mu$ decays of each sign for a stored muon energy of 10,20 and $30 \mathrm{GeV}$. The appearance of electrons muons is enhanced(suppressed) by matter effects for decaying $\mu^{-}\left(\mu^{+}\right)$beams.

In the case of $\mu^{+}$beams, process 1) involves neutrinos, so the depletion of electron events from the beam is matter-enhanced, while process 2 ) is suppressed. The net effect is a smaller number of observed events with leading electrons with respect to the expectations, as can be seen in Tables 3 and 1 .

In conclusion, even without charge discrimination, the matter effect asymmetry is visible between leading electron events from $\mu^{-}$and from $\mu^{+}$decays.

\subsection{Events with no leading leptons}

Events with no leading electrons or muons will be used to study the $\nu_{\mu} \rightarrow \nu_{\tau}$ oscillations. These events can be produced in

1. neutral current processes

2. hadronic $\tau$ decays

$$
P_{\nu_{\mu} \rightarrow \nu_{\tau}} \times B R(\tau \rightarrow \text { hadrons }) \times \Phi_{\nu_{\mu}}+P_{\bar{\nu}_{e} \rightarrow \bar{\nu}_{\tau}} \times B R(\tau \rightarrow \text { hadrons }) \times \Phi_{\bar{\nu}_{e}}
$$

The neutral current processes do not depend on the oscillations, so there is always an excess of events in this class with respect to the expectation due to the hadronic $\tau$ decays. The total number of events for these two categories is shown in Table 4 , for both $\mu^{-}$ and $\mu^{+}$beams. The large excess of neutral current like events will be a clear signal for conversion to $\nu_{\tau}$ flavor. A $\nu_{\mu}$ disappearance signal not compensated by these events would be a clear sign for a different type of conversion, like for example to a sterile neutrino.

\begin{tabular}{ccccc}
\hline$E_{\mu}(\mathrm{GeV})$ & \multicolumn{2}{c}{$\mu^{-}$beam } & \multicolumn{2}{c}{$\mu^{+}$beam } \\
& $\mathrm{NC}$ & $\tau \rightarrow$ hadrons & $\mathrm{NC}$ & $\tau \rightarrow$ hadrons \\
\hline \hline 10. & 196 & 21 & 173 & 25 \\
20. & 3534 & 1383 & 3168 & 847 \\
30. & 16061 & 5180 & 14553 & 2771 \\
\hline
\end{tabular}

Table 4: Contributions to events with no leading leptons normalized to $10^{21} \mu$ decays of each sign for a stored muon energy of 10, 20 and $30 \mathrm{GeV}$. The appearance of neutral current like events due to hadronic tau decays is clearly seen. 
Event energy spectra

In the previous section, we have compared the expected number of events of four classes of events and shown how they are affected by the presence of neutrino oscillations and by matter effects. More information on the nature of the oscillations can be extracted by using the total visible energy of an event, which is a measure for the incoming neutrino energy. Figure 3 shows the energy spectra of the four classes of events defined in section 5 for decays of $30 \mathrm{GeV}$ muons. The four left hand side plots correspond to $\mu^{-}$decays in the storage ring, while the four right hand side plots are for $\mu^{+}$decays. The dotted and full lines refer to the predicted distributions without and with oscillations with $\Delta m^{2}=3 \times 10^{-3} \mathrm{eV}^{2}$.

The spectacular disappearance of right-sign muon is visible in Figure 3 plots b). The enhancement(suppression) due to matter effects is clearly visible in the plots c) which contain the opposite-sign muon events. The apperance of tau can be directly observed as an excess of neutral current like events (plots d).

A fit to the observed energy distributions and rates of the right-sign muon events would allow the precise determination of the parameters $\Delta m^{2}$ and $\phi$ governing the oscillation.

The opposite-sign muons will directly probe the $\theta$ angle and will test the MSW effect. An indirect measurement of the $\Delta m^{2}$ parameter can be extracted from the energy spectrum of opposite-sign muons since the position of the peak of the distribution is directly related to the resonance energy, which is a function of $\Delta m^{2}$ (see section 4 eq. 8).

\section{$7 \quad$ Conclusions}

The observation of the MSW resonance by a terrestrial experiment for the parameters suggested by the atmospheric neutrino experiments is an integral part of the phenomenology of neutrino oscillations. Although hardly possible with the present generation of long baseline beams, it becomes possible in a synergic approach to the development of the novel muon colliders. The appearance of the MSW resonance will be truly spectacular. The enhancement(suppression) for neutrinos(anti-neutrinos) will be directly selected by the charge of the muons in the storage ring. The statistics in a 10 kton detector obtained from muon beams with the preliminary parameters needed for muon colliders will be sufficient to accurately measure the resonance parameters, providing a powerful tool to determine the relevant mass squared difference $\Delta m^{2}$. The large statistics at high energy will also allow the study of all neutrino flavor oscillations, including the $\nu_{\tau}$ apperance, and will provide an accurate determination of the $\Delta m^{2}$ and the mixing angles.

\section{Acknowledgments}

We are indebted to R. Palmer for useful discussions on the AGS at BNL.

\section{References}

[1] L. Wolfenstein, Phys. Rev. D17, 2369 (1978); Phys. Rev. D 20, 2634 (1979); Mikheyev and Smirnov, Sov.J.Nucl.Phys. 42, 913 (1986).

[2] J.N. Bahcall, P.I. Krastev and A.Y. Smirnov, Phys. Rev. D58, 096016 (1998) hepph/9807216 and references therein.

[3] Y. Fukuda et al. [Super-Kamiokande Collaboration], Phys. Rev. Lett. 81, 1562 (1998). 
[4] K.S. Hirata et al., Phys. Lett. B 205, 416 (1988); Phys. Lett. B 280, 146 (1992). Y. Fukuda et al., Phys. Lett. B 335, 237 (1994).

R. Becker-Szendy et al., (IMB Collab.), Phys. Rev. D 46, 3720 (1992).

W.W. Allison et al. [Soudan-2 Collaboration], Phys. Lett. B449, 137 (1999) hepex/9901024.

F. Ronga et al. [MACRO Collaboration], "Atmospheric neutrino induced muons in the MACRO detector," hep-ex/9810008.

[5] P.Lipari, hep-ph/9903481.

[6] C.M. Ankenbrandt et al., "Status of muon collider research and development and future plans," FERMILAB-PUB-98-179.

[7] S. Geer, Phys. Rev. D 57, 6989 (1998).

[8] A.Bueno, M.Campanelli, A.Rubbia "A medium baseline search for $\nu_{\mu} \rightarrow \nu_{e}$ oscillations at a $\nu$ beam from muon decays" hep-ph/9809252 CERN-EP/98-140 accepted by IJMP.

A. De Rujula, M.B. Gavela and P. Hernandez, Nucl. Phys. B547 (1999) 21 hep$\mathrm{ph} / 9811390$.

[9] A.Bueno, M.Campanelli, A.Rubbia "Long baseline neutrino oscillation disappearance search using a $\nu$ beam from muon decays" hep-ph/9808485 ETHZ-IPP-98-05 accepted by IJMP.

[10] J. Bahcall and P. Krastev, Phys. Rev. C 56, 2839 (1997), hep-ph/9706239.

[11] Robert Palmer and Thomas Roser, private communications.

[12] see e.g. G.L. Fogli, E. Lisi, and G. Scioscia, Phys. Rev. D 52, 5334 (1995).

[13] M. Apollonio et al. [CHOOZ Collaboration], Phys. Lett. B420, 397 (1998), hepex/9711002.

[14] M.Campanelli, A.Bueno, A.Rubbia, "Three-family oscillations using neutrinos from muon beams at very long baseline", ICARUS-TM-99/13.

[15] ICARUS collaboration, "ICARUS-Like Technology for Long Baseline Neutrino Oscillations", CERN/SPSC/98-33 \& M620, 1998 and references therein. 


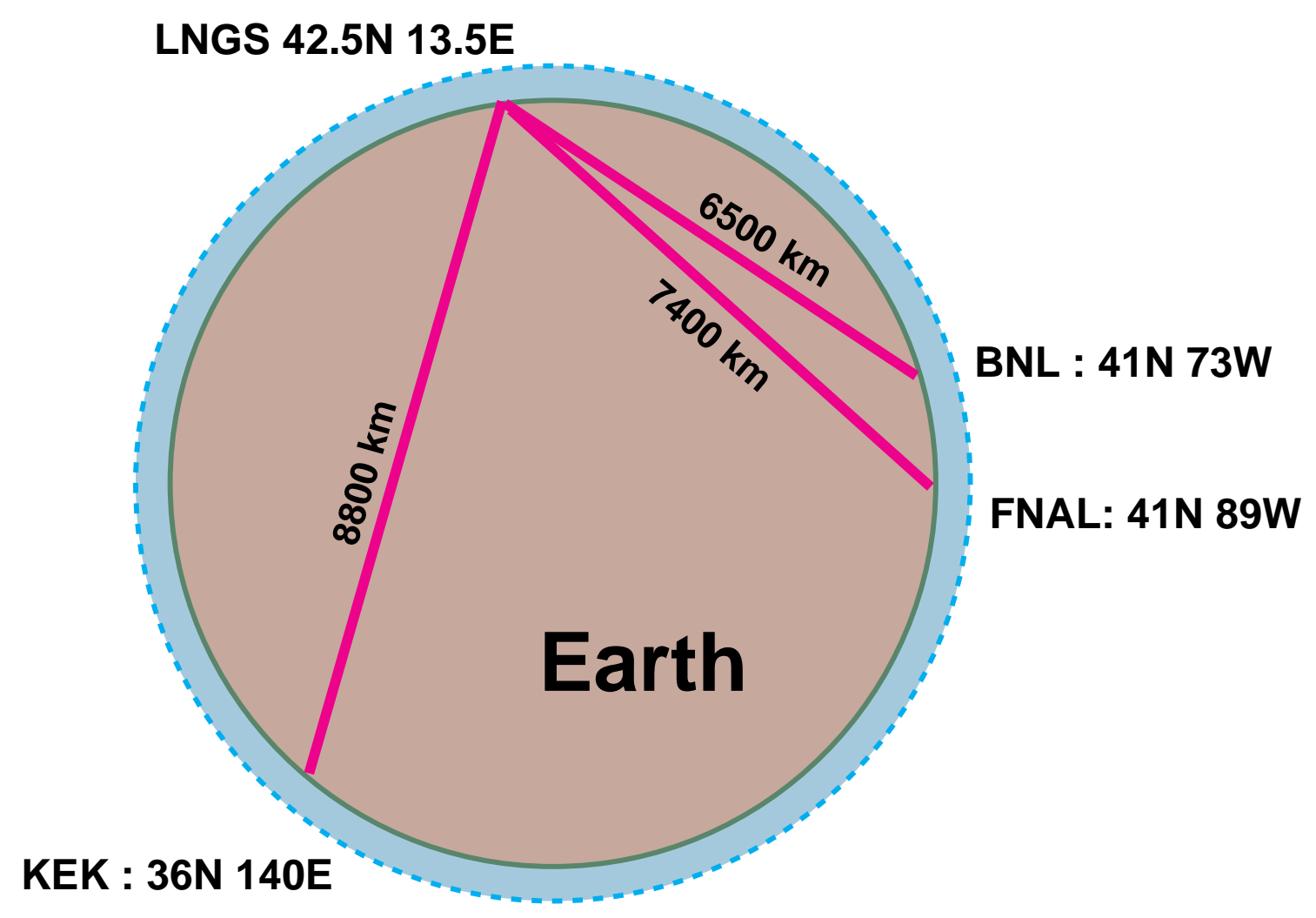

Figure 1: Possible very long baselines across the Earth (seen from above the North pole). 

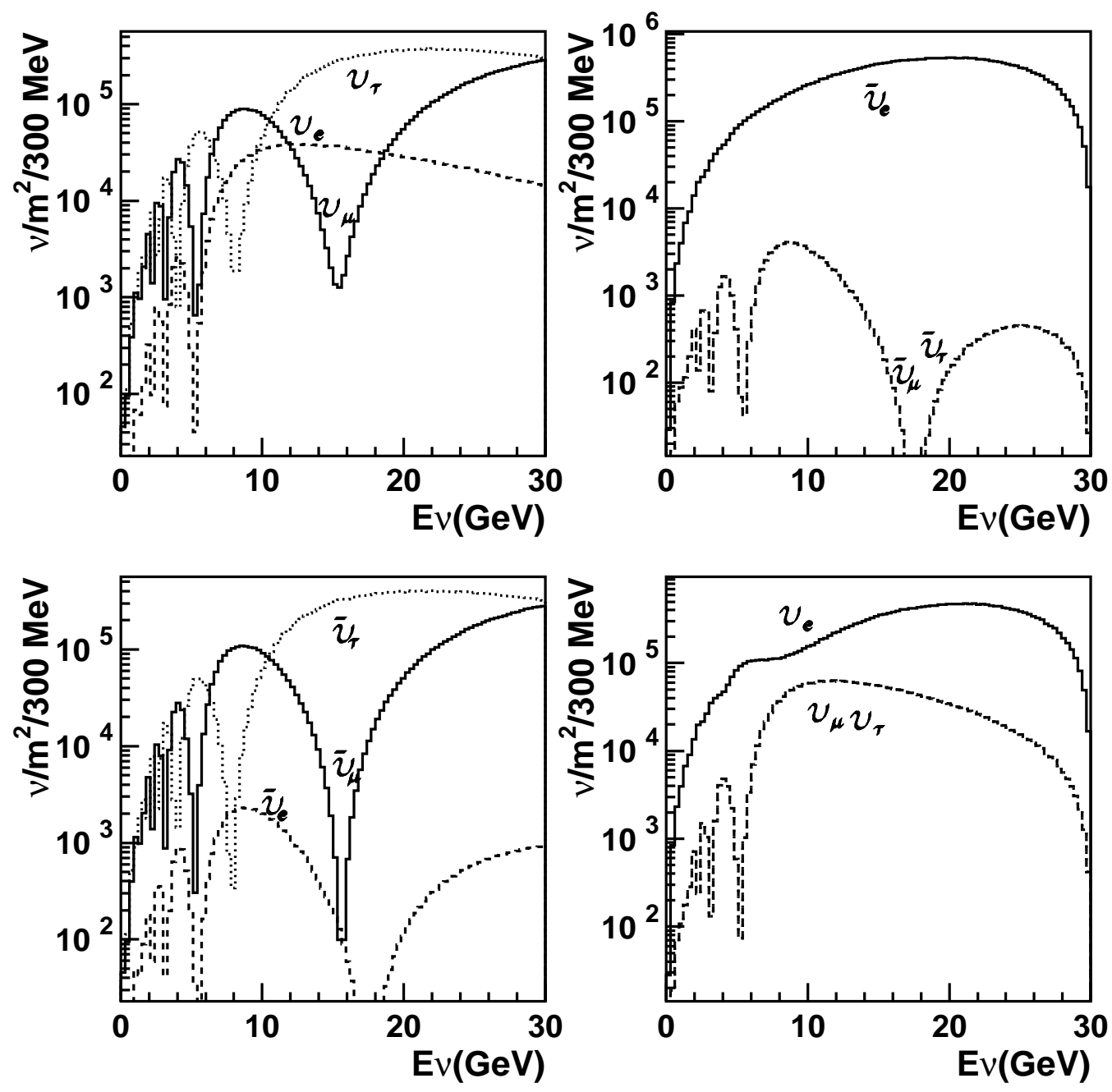

Figure 2: The oscillated neutrino fluxes reaching the detector per $m^{2}$ normalized to $10^{21}$ muon decays. The oscillation probability is given for three-family mixing with $\Delta m^{2}=$ $3 \times 10^{-3} \mathrm{eV}^{2}, \sin ^{2} \phi=0.5, \sin ^{2} \theta=0.025$. The two upper plots refer to decays of $30 \mathrm{GeV}$ $\mu^{-}$, the two lower to decays of $\mu^{+}$. The matter enhancement(suppression) is clearly visible for the $\nu_{e}\left(\bar{\nu}_{e}\right)$ cases. The $\nu_{\mu}\left(\bar{\nu}_{\mu}\right)$ fluxes are largely suppressed with a spectacular "hole" in the spectrum due to maximal oscillation to $\nu_{\tau}$. The $\nu_{e} \rightarrow \nu_{\mu}$ and $\nu_{e} \rightarrow \nu_{\tau}\left(\bar{\nu}_{e} \rightarrow \bar{\nu}_{\mu}\right.$ and $\left.\bar{\nu}_{e} \rightarrow \bar{\nu}_{\tau}\right)$ contributions are also visible. 

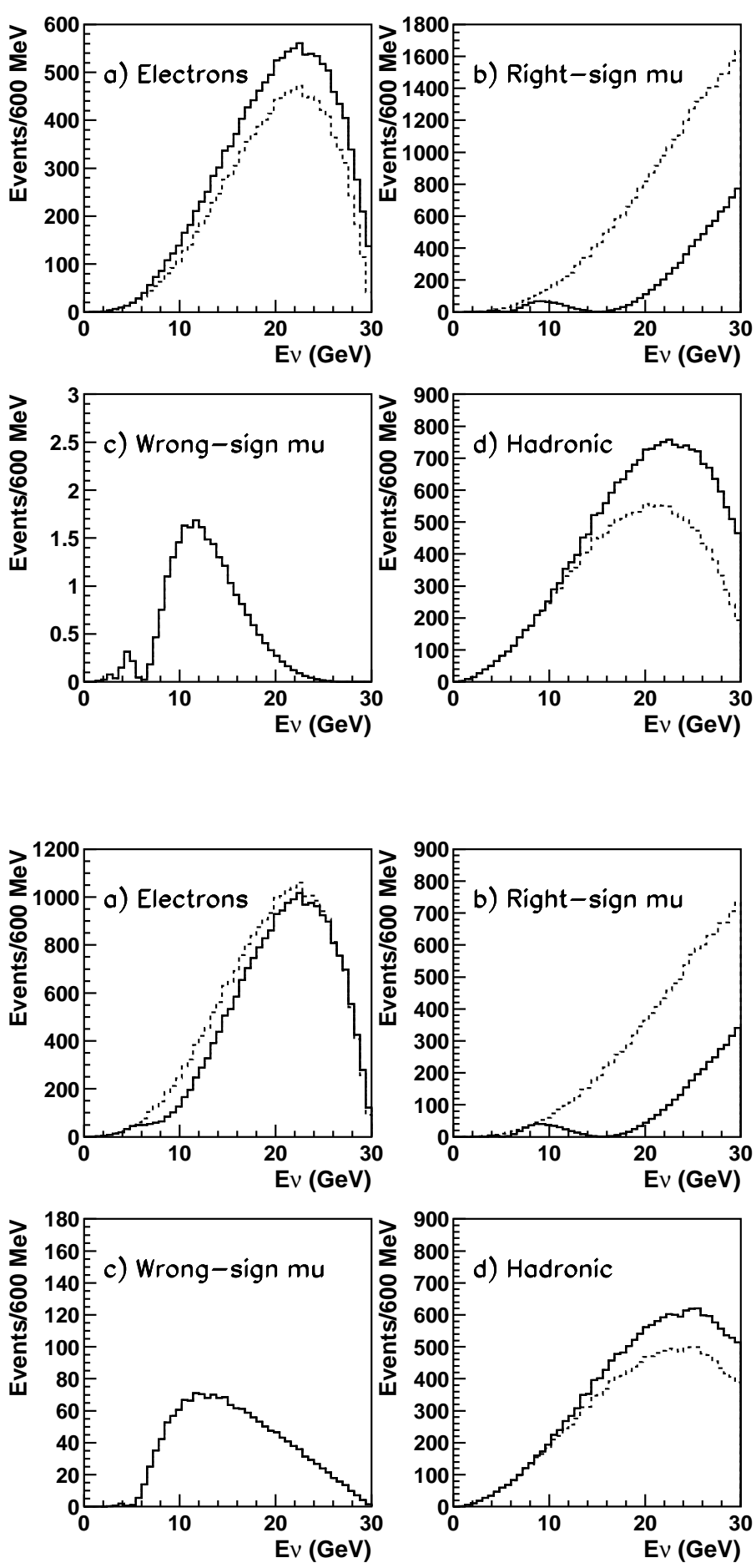

Figure 3: Predicted event energy spectrum of the four classes of events a) with leading electron or positron, b) with leading right-sign muons, c) with leading opposite sign muons d) events with no leading leptons. Full line: spectra with oscillations; dashed line: spectra without oscillations. The four upper plots are for $10^{21} \mu^{-}$decays and the four lower plots are for $10^{21} \mu^{+}$decays. The muon energy is $30 \mathrm{GeV}$ and the target mass is $10 \mathrm{kton}$. The spectacular disappearance of right-sign muon is visible in plots b). The enhancement(suppression) due to matter effects is clearly visible in the plots c) which contain the opposite-sign muon events. The apperance of tau can be directly observed as an excess of neutral current like events (plots d). 